\title{
Can (imported) Knowledge Change Systems? Understanding the Dynamics of Behaviour Change
}

\begin{abstract}
Chapter 4 reviews a wide range of theoretical material in search of effective explanations for the intervention failures we have observed, and the resulting impact on the SVP volunteer deployment model. The step from training through learning to individual behaviour change was not fully understood conceptually or in terms of operational dynamics. We have learnt that knowledge mobilisation does not automatically derive from learning; knowledge in itself may be empowering or disempowering. Knowledge mobilisation is highly contextualised and needs to be understood within the frame of wider human resource management systems. Chapter 4 presents a critique of behavioural science theories, which have essentialising tendencies, and proposes ideas from evolutionary economics around 'imagined realities' and action planning which help to understand the contextual dynamics impacting on systems change.
\end{abstract}

Keywords Motivation - Behaviour change - Imagined realities - Action planning

\section{INTRODUCTION: THEORETICAL INSIGHTS from Diverse Disciplines}

Chapter 2 discussed the importance of volunteer roles to an understanding of the outcomes associated with professional voluntarism focusing, in particular, on the risks and systems damage associated 
with service delivery and labour substitution. Drawing on theoretical insights and grounded research, the SVP operationalised the principle of 'co-presence' to protect against lone working and labour substitution and foster the kinds of relationships conducive to optimal knowledge exchange and co-learning.

Recognition of the externality effects (unintended consequences) associated with service delivery (by volunteers) has led to an increasing emphasis on 'capacity-building' amongst key stakeholders and funders. And, in most cases, this has been interpreted to imply that professional volunteers should be primarily engaged in training/education and knowledge transfer activities. Chapter 3 reported evidence of the role that professional volunteers (in carefully structured programmes) can play as 'boundary spanners' and knowledge brokers. However, whilst transferring knowledge is evidently possible, it also reflected on the failure of these approaches to stimulate and sustain lasting and effective systems change. Put simply, the knowledge is being shared but the impact is minimal and short-lived, rarely extending beyond volunteer stays.

Chapter 4 addresses the 'why' question: why is systems change so elusive even in an environment of carefully managed knowledge transfer, translation and exchange? These concerns, drawing directly on a wealth of lived/empirical evidence, stimulated the authors to search for theoretical insights capable of throwing light on the dynamics involved and informing future interventions. It is highly unusual to place a chapter of this nature towards the end of a book. The fact that it is where it is reflects the essentially iterative quality of our research journey and the inter-weaving of theory with empirical work. In this case, it is the empirical work that led to our search for new theoretical ideas to help us to understand our findings as they emerged and then, in turn, stimulate new empirical questions.

Theories exist to facilitate understanding and explain social phenomenon. Sadly, they are (almost always) associated with the construction of concepts and language that render them inaccessible to anyone outside the narrow disciplinary confines responsible for their development. Disciplinary silos tend to generate exclusive language that restricts the very objectives of theorisation (understanding) to the point at which small groups of people are essentially communicating only with each other. This chapter seeks to interpret a complex (if not comprehensive) range of theory to assess the potential for more holistic, multi-disciplinary, insights into the dynamics of health worker behaviour. 


\section{Our 'Journey' through the Disciplines}

As noted before, and discussed in more detail in Chapter 2, the whole process and more specifically, the SVP project, was profoundly shaped by theoretical work coming primarily from geography but drawing on other disciplines (business and education). Theories of knowledge and the conditions shaping its mobility across international space informed the development of the volunteer agreements, associated partnership agreements (Memoranda of Understanding with Ugandan partners) and, most notably in operational terms, the co-presence principle that lies at the heart of the SVP. As our evaluation of the SVP led us to question the efficacy and sustainability of interventions involving professional volunteers in terms of longer-lasting systems change and patient outcomes, we began to search for other ways of understanding these complex change/ inertia dynamics.

In the first instance, perhaps because our search was (somewhat unwittingly) influenced by the clinical focus of our Ugandan work (i.e. on improving maternal and newborn health) it took us directly to a burgeoning literature associated with the disciplines of behavioural psychology, implementation science and evidence-based medicine. We refer loosely to this collection of theories as 'behavioural science' (BehSci). In some respects, these theories share common ground with classical economic theory centred on the concept of the individual decision maker underpinned by ostensibly quantitative 'data'.

This corpus of work offered immediate and poignant insights into the relationship between knowledge/learning and individual behaviour change. And the work had a major influence on our thinking, encouraging us to conceptualise the 'problem' as one relating to behaviour change. However, as we explored the work in greater depth we began to realise that the concepts used, the approaches to the theorisation of knowledge and the associated privileging of quasi-scientific methods coupled with a profound emphasis on the individual failed to resonate comfortably with our research experiences and observations in Uganda. The BehSci literature examined the relationship between individual 'capabilities' and motivation. Ackers' background in socio-legal research and the supra-national impacts associated with European law reminded us of very different conceptualisations of 'capabilities' and encouraged us to revisit socio-legal theory, which itself draws heavily on social philosophy. This work takes us beyond individuals and organisations to more structural and over-arching elements of context 
and, specifically, the role that legal systems and social policy (sometimes referred to as 'soft law') play in shaping individual and organisational behaviour. Of particular interest here is the emphasis on agency and empowerment - on individuals as active citizens in complex and 'tiered' or 'nested' relationships with the State rather than as isolated and passive victims. This literature, which is primarily theoretical, also draws interesting parallels with philosophical concepts of positive freedoms (enabling social rights) sometimes known as the 'freedom to' (do something) as opposed to the more familiar 'freedom from' (laws protecting citizens from legally sanctioned behaviour). We shall refer to this very loosely as the 'capabilities approach' (CA).

The emphasis on employment in Barbard's socio-legal paper (2001) sparked an interest in another flourishing area of research focused on human resource dynamics. There is a considerable literature applying human resource management (HRM) ideas and theories to an understanding of health worker motivation in high- and low-resource settings. Interestingly, this literature focuses primarily on the organisational context and tends to be characterised by more qualitative (case study) approaches. Our first reaction to this body of work was to identify with the emphasis on organisational dynamics and the deeply contextual quality of these - something that is present but remains largely peripheral in the behavioural science work. And also to nudge us into a significant if obvious realisation that health worker motivation is fundamentally not a clinical question - so there is little surprise that 'evidence-based medicine' (with its origins in clinical trials) fails to capture adequately the dynamics involved and perhaps lacks the tools to do so.

Somewhat by coincidence $\left(\right.$ serendipity ${ }^{1}$ ) we stumbled upon another approach espoused by evolutionary economists but using language and ideas that, to us at least, seemed remarkably different to classical economics. This fundamentally theoretical work uses rather different concepts to discuss essentially the same challenge: how to mobilise knowledge in order to optimise outcomes.

We shall group the work reviewed here loosely under the heading of 'Evolutionary Economics' (EE). It will perhaps come as no surprise that this group of theories utilising concepts including 'imagined realities' and 'innovative intentionality' (as with the socio-legal ideas mentioned earlier) are unapologetically theoretical and largely untested in empirical contexts. It is perhaps for this reason that they are able to engage so luxuriously with context and imagination. 
This chapter reviews the work referred to earlier. The aim here is to draw on our existing grounded empirical knowledge and experience to identify approaches and concepts that appear to be most relevant and offer greatest potential to an understanding of the role of the individual health worker in the advancement of health systems change in the Ugandan public sector. We have tried, wherever possible, in order to optimise engagement and inter-disciplinary communication to avoid excessive use of complex terminology and referencing. And we apologise in advance if this has failed or results in an over-simplification.

\section{Insights from the Behavioural Sciences (BehSci)}

The Behavioural Science work reviewed here shares three key premises. First, that 'improving the implementation of evidence-based practice and public health depends on behaviour change' (Michie et al. 2011: 1). This focuses attention on the importance of individual behaviour change. BehSci theorists contend that, 'Progress in tackling today's major health and healthcare problems requires changes in the behaviour $[\ldots]$ of healthcare professionals' (Michie et al. 2009: 1). Second, and this is where the attraction of the theory first sparked our interest, it explicitly recognises that knowledge in itself cannot change behaviour:

For most health behaviours [ ...] knowledge is not an important source of variance (Cane et al. 2012: 15).

This implies that knowledge in itself will not automatically generate capacity-building or systems change. To understand variance we need to look elsewhere. Finally, 'behaviour change techniques' form part of what proponents describe as the 'science and technology of behaviour change' (Michie et al. 2011:2). This underlines the epistemological ${ }^{2}$ underpinnings of BehSci theories; the privileging of 'scientific' methods and the inference (by reference to 'techniques') that change lies at individual level and that interventions targeted at that level can work.

The behavioural science literature reviewed here is applied to two rather different phenomena: first (and perhaps this is where the more clinical orientation derives from), it is used in public health contexts to try to improve patient behaviour (smoking cessation, addiction or healthy eating, for example). The same ideas are then applied to a rather different context; namely, the behaviour of healthcare professionals. 
And, in this sphere, they are applied to more readily 'measurable' specific clinical behaviours (such as antibiotic prescribing).

Evidence that such interventions have largely failed to generate the intended impact have raised concerns that impact is limited not because of the quality of the knowledge transferred per se (or knowledge transfer mechanisms) but because the 'behaviour change intervention' is neither evidence-based nor theoretically informed (i.e. linked to a comprehensive model of behaviour). Michie et al. argue that interventions need to be designed so as to 'bring healthcare professionals into line with evidencebased practice' (2009: 1). A systematic review of published interventions by Michie et al. leads them to conclude that researchers were 'less confident about being able to replicate behavioural interventions compared with pharmacological interventions' (2009: 2). Attributing this to low levels of investment in research in this area (in comparison with pharmacology) rather than any more fundamental differences involved in comparing the relationships that humans have with inanimate objects (such as a statin or aspirin) with those more inter-subjective relationships that humans have with each other, they propose the development of a science or 'technology' of behaviour change to support accurate replication.

Whilst we would absolutely support their argument in favour of strengthening the reporting of observational studies, we would challenge the appropriateness of extending existing guidelines for the reporting of pharmacological research to analyses of health worker behaviour given the complex inter-subjectivity that the latter involves. Perhaps it is the language used and its reference to 'ingredients' and the 'science of behaviour' that makes us cautious about the applicability of these ideas to Uganda health workers:

Just as medicines are described in detail in the British National Formulary (BNF) we need a parsimonious list (nomenclature) of conceptually distinct and defined techniques, with labels that can be reliably used in reporting interventions across discipline and country (2009: 4).

Working with another group of authors, Michie proposed the now familiar model of the 'Behaviour Change Wheel' as a 'method for characterising and designing behaviour change interventions' (2011: 1). The 'COM-B' ${ }^{3}$ model is designed in the first instance to predict patients' responses to public health interventions around smoking cessation and obesity in England. It defines behaviour change interventions as those 'designed to 
change specified behaviour patterns' signalling a focus on individuals (as patients and healthcare workers). It is perhaps interesting to note that the emphasis at this point is not on understanding behaviour but changing it. Subsequent reference to 'behaviour change techniques' reinforce this assumption: dysfunctional individuals lie at the heart of the problem. Michie et al. outline the methods used to develop their model grounded in 'systematic review'. The concept of systematic review is very much linked to the evidence-based medicine movement and derives from methods developed through the Cochrane Collaboration. ${ }^{4}$ The NHS Centre for Reviews and Dissemination (CRD) defines a systematic review as, 'a review of the evidence on a clearly formulated question that uses systematic and explicit methods to identify, select and critically appraise relevant primary research, and to extract and analyse data from the studies that are included in the review' (CRD 2001: 3)

There is no scope here to discuss and critique the relative merits of systematic reviews and their claims to objectivity. Certainly their value and approach in understanding clinical drugs trials is undisputed. For us, as social scientists more accustomed to the concept of 'literature' or 'research review', they present a certain narrowness in focus that may risk excluding highly relevant and innovative multi-disciplinary knowledge and/or grey literature (Benzies et al. 2006). The emphasis in systematic review processes on the 'clearly formulated question' tends to lead to a funnelling approach, progressively narrowing inquiry to an ever-smaller group of highly similar studies. Whilst this approach may form a key component of the comparability criteria necessary in clinically oriented systematic reviews (to compare like with like and reduce extraneous 'noise'), it lies in some tension with more expansive and exploratory 'searchlight' or horizon-scanning approaches to literature review explicitly seeking new knowledge and innovative insights (as represented in this chapter).

Furthermore, the 'quality appraisal' component of systematic reviews is based on the premise that research can be ranked according to its quality, reliability and replicability. In practice, this involves a weighting process based on metrics to assess 'the rigor of the research methodology' (Jones et al. 2013: 3) effectively privileging quasi-experimental techniques. At the apex of this epistemological hierarchy (Levels 1 and 2) lies the randomised controlled trial (developed from clinical research) and, at the base, opinion pieces. Qualitative research receives no specific mention in this schema but presumably falls within the generic category (Level 3) of 'non-randomised, 
controlled or cohort studies, case series, case controlled studies or cross-sectional studies' (Benzies et al. 2006). ${ }^{5}$ We are also concerned that the notion of 'validated' tools appears to ignore the fact that the validation process may take place in a quite distinct context (UKor US-based addiction studies, for example) and is then applied to a quite different context (health worker behaviour in low-resource settings).

Just as the systematic review process employed in this material has the tendency to encourage blinkered approaches and restrict the exploration of new knowledge, their approach to theory has the same effect. Cane et al. contend that, 'behaviour change interventions informed by theory are more effective than those that are not' (2012: 1). Certainly, a robust theoretically informed approach promises greater chance of success than what are often ad hoc, uncoordinated and often conflicting interventions. And 'opinion pieces' would generally not be published in peer-reviewed journals in the social sciences.

However, the 'comprehensive theoretical model' presented by Cane et al. involved engaging with 18 psychological theorists and 30 health psychologists (2012:2). They were in effect talking to each other. The important point here is that concerns (that we share) about the lack of theoretical foundations are interpreted within such myopic disciplinary lenses. Interestingly, the authors propose that their integrative 'Theoretical Domains Framework' (TDF) developed through work with the aforementioned groups will 'make theory more accessible to, and usable by, other disciplines' (2012:2). ${ }^{6}$ This approach has parallels with surrogacy: the genetic map is intact and 'given' and the surrogate discipline is then able to apply it but not to re-combine it with its own knowledge to alter the essential architecture.

Having discussed some of the concerns underpinning the generation of behaviour change models, we now turn to assess the potential value of the models themselves in terms of supporting our understanding of the failure of professional voluntarism to stimulate sustainable health sector reform in Uganda.

The 'Behaviour Change Wheel' is described by Michie et al. as a 'potentially elegant way of representing the necessary conditions for a volitional behaviour to occur' (2011: 4). In this 'behaviour system', capability, opportunity and motivation interact to generate behaviour, which, in turn, influences these core components. Or, put more simply, individual behaviour change is the sum of an individual's 


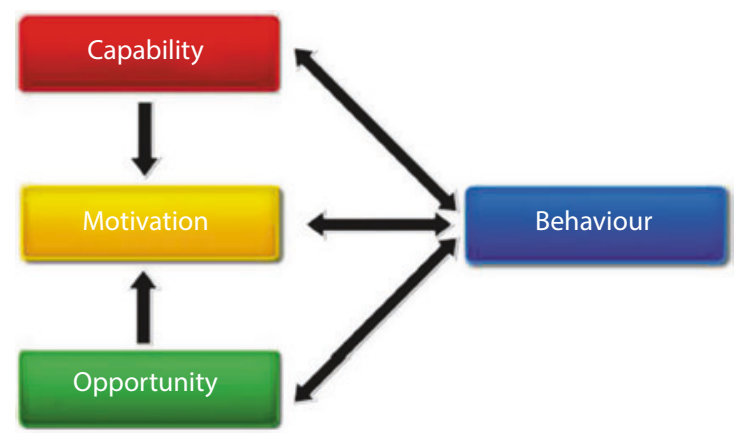

Fig. 4.1 The COM-B system (Source: Michie et al. 2011.) All rights reserved, used with permission.

capability (their knowledge and skills); the opportunities they have to utilise those skills and, critically, their motivation to do so. Much CME interventions, as discussed in the previous chapter, focus attention only on improving individual capability. These relationships are illustrated in (Fig. 4.1).

Whilst Michie et al. argue that this system gives no priority to individuals, groups or environmental perspectives - or intra-psychic or external factors - in controlling behaviour the emphasis in practice is very much on the individual. Capability in this model has a specific, individualised, definition - namely, 'the individual's psychological and physical capacity to engage in the behaviour' including knowledge and skills (2011: 4). Motivation is also defined in individual terms as 'all those brain processes that energise and direct behaviour' (2011: 4). ${ }^{7}$ Finally, opportunity is 'all those factors that lie outside the individual that make behaviour possible or prompt it'. Michie et al. argue that this framework 'incorporates context very naturally [through] the "opportunity component"' (2011: 8). It is clear then that both capability and motivation are internal to the individual in this model.

The simple elegance of this model is what attracted us to it in the first instance. It immediately captures the errors behind the 'training fetishism' described in Chapter 3 and helps to explain why endlessly training Ugandan health workers in neonatal resuscitation or triage fails to translate into sustained behaviour change and improved patient outcomes. The concept of motivation has an immediate resonance too with ethnographic observations and the narratives of volunteer interviews. Certainly, at face value, the 
majority of employees in the public healthcare system in Uganda give the appearance of and readily describe themselves as 'demotivated'. And, it is all too obvious that the lack of opportunities to utilise skills due to lack of equipment, drugs or stationary inevitably leads to both a lack of motivation to learn and also a frustrating inability to exercise newfound skills. The logic of this model 'explains' why the deployment of professional volunteers solely to teach and train (transmit knowledge) is failing to impact systems.

The COM-B system outlined earlier is positioned at the heart of a 'wheel' which identifies potential 'intervention functions' and policy categories that may or may not form part of an intervention depending on the analysis of the situation and where the problem lies (Fig. 4.2).

In a further development, Cane et al. map their 'theoretical domains framework' onto Michie's wheel suggesting that the two approaches work together well in informing interventions (Cane et al. 2012: 12) (Table 4.1).

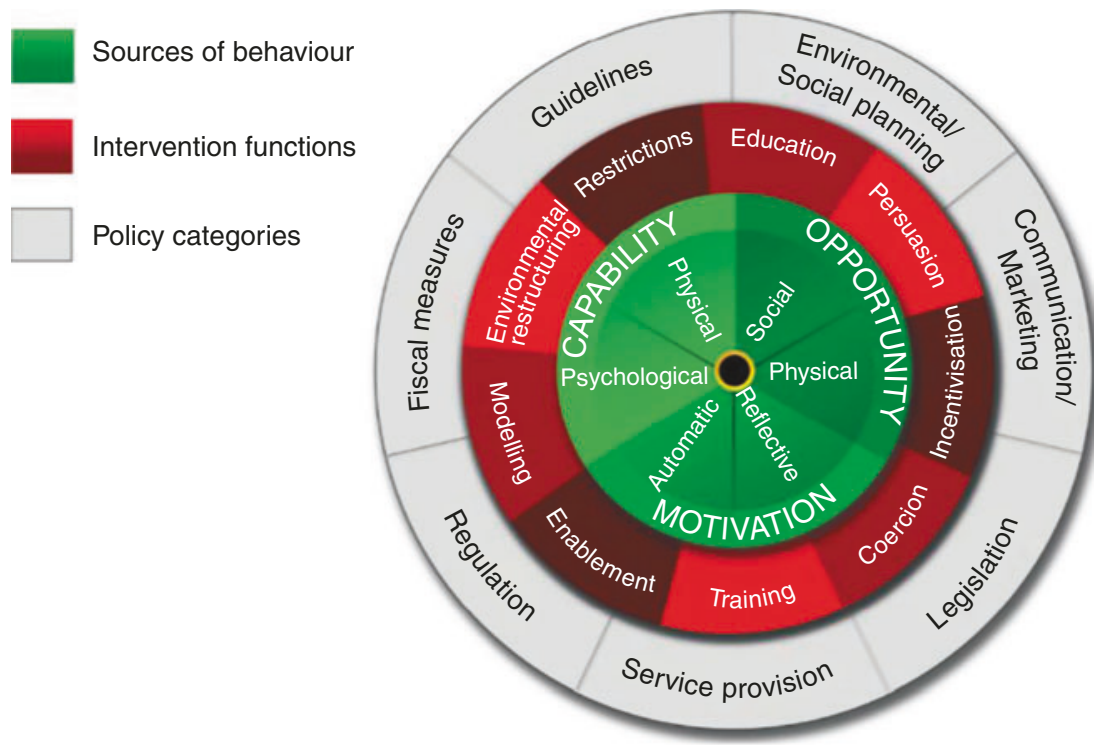

Fig. 4.2 The Behaviour Change Wheel (Source: Michie et al. 2011: 1.) All rights reserved, used with permission. 
Table 4.1 Mapping of the Behaviour Change Wheel's COM-B system to the TDF domains

\begin{tabular}{lll}
\hline COM-B & & TDF domain \\
\hline Capability & Psychological & Knowledge \\
& & Skills \\
& Memory, attention and decision processes \\
& & Behavioural regulation \\
& Physical & Skills \\
Opportunity & Social & Social influences \\
& Physical & Environmental context and resources \\
Motivation & Reflective & Social/professional role \& Identity \\
& & Beliefs about capabilities \\
& & Optimism \\
& & Beliefs about consequences \\
& & Intensions \\
& & Goals \\
& Automatic & Social/professional role \& identity \\
& & Optimism \\
& & Reinforcement \\
& & Emotion
\end{tabular}

Source: Cane et al. 2012: 15. All rights reserved, used with permission.

Once the convenience of the model had sunk in and we began to consider more carefully how this related to our own experience and knowledge of volunteer engagement in Uganda, concerns emerged about its ability to capture the complexity of the real-world context. The explicit individualism of this model and the assumption that health systems are a collection of individuals rather than complex social and relational structures raised some concerns. In many ways, this is reminiscent of Margaret Thatcher's famous declaration that, 'There is no such thing as society. There are individual men and women and there are families' (Interview in Women's Own 1987). ${ }^{8}$ The implied emphasis here is on dysfunctional individuals and the 'techniques' that can be devised to render them functional. We became particularly interested in the concept of 'capabilities' used here as something internal and reducible to an individual: a 'given'.

Certainly our understanding of health workers had made us very aware that the same person could show a high level of motivation and capability in different contexts. We have been involved in hosting many Ugandan 
health workers in the UK through the Commonwealth Professional Fellowship Scheme and with few exceptions in this context there is no evidence that they lack skills or motivation. Equally, staff members working on a private ward in a public hospital or in private clinics appear to work far more effectively than they do on the public wards. Does this suggest that capability is not an individual trait as such but contextual and also that 'opportunity' is everything?

\section{Perspectives from Socio-Legal Studies and Social Philosophy (CA)}

It was at this point that we reviewed some of the work on capabilities from the perspective of socio-legal studies, which itself draws on social philosophy and citizenship studies. This research would most certainly not be picked up in a systematic review. We have called this group of theories the 'capability approach' (CA). Once again, this literature uses complex concepts and language, potentially limiting accessibility and opportunities for holistic multi-disciplinary thinking. Barbard's paper addresses 'capabilities' from the perspective of the development of the European Union and its associated legal institutions. It is concerned primarily with the conditions necessary for the realisation of the European Council's strategic goal of becoming 'the most competitive and dynamic knowledge-based economy in the world capable of sustainable economic growth with more and better jobs and greater social cohesion' (2001: 464).

At first sight we may dismiss this work as somewhat peripheral to our concerns (with professional voluntarism and health systems in lowresource settings). The language used is more up-beat; less about 'problems' and more about knowledge for foresight and innovation. However, the immediate link with 'investing in people and combating social exclusion' lies at its heart. What initially interested us was the quite different approach to the concept of capabilities. Here they are not reduced to intrinsic or raw attributes of individuals but linked to concepts of agency and 'proactive security' (2001: 467). Barbard first critiques neoclassical economic theories which conceptualise what they call 'individual self-sufficiency' as a function of individual endowments, 'consisting of genetically-inherited capacities for work, and the financial and other resources made available to them' (2001: 465). In this model 
then outcomes are a function of inputs (individual innate ability) and resources. Drawing on the work of Sen (1999), Barbard proposes the addition of other functions identifying capability as 'a kind of freedom: the substantive freedom to achieve alternative functioning combinations' (citing Sen 1999: 75):

Mobilising the economic potential of individuals is not simply a process of providing them with the necessary financial resources to exploit their endowments. Rather, the institutional framework of the market has to be examined in order to establish how far it facilitates or constrains the potential of individuals to achieve their desired economic functionings (p. 466).

Put simply, mobilising the economic potential of individuals is not simply a combination of innate endowments (intelligence) and resources (intellectual and physical). Capabilities in this approach are less reductionist and more composite than in BehSci theories: capabilities are not knowledge as such but mobilise (convert) knowledge. The practical example Barbard gives to illustrate her point helps to clarify the argument. Whilst sex discrimination law in the EU formally sanctions overt discrimination against female employees (a negative freedom or freedom 'from' discrimination), it is the more positive form of 'freedom to' sometimes referred to as soft law or policy, in the form of subsidised childcare or paid maternity leave, for example, that alters incentive structures enabling women to make personal investments in skills and training. Achieving gender equality demands both approaches.

For Barbard, individual behaviour (if we can phrase it that way) requires an assessment of how far the labour market (or the 'environment' in the COM-B model) facilitates or constrains the potential of individuals to utilise their knowledge and abilities. Barbard uses the concept of 'institutionalised capabilities' to describe the infrastructure of social entitlements (social rights and associated support) that facilitate and enable active participation. For Barbard, this infrastructure is not a luxury but a fundamental precondition of a functioning labour market providing the necessary incentive structures (proactive security) to enable and facilitate individual behaviour change. Indeed, the logic of this model is that, in the absence of institutionalised capabilities, investment in training is ineffective and wasteful: training inputs focused on generating changes in aggregate individual behaviour will not work. As critics of market liberalism have argued, society is more than a sum of its parts. And people need rights and resources to engage with knowledge. 
Can this model be applied to the very different context of lowresource settings and the specific institutional context of health systems? We cannot see why not. Barbard's conclusion that the European Social Model needs to combine social rights, economic commitment with highquality employment opportunities and industrial relations (dispensing with the idea that economic growth or institutional change can be achieved with a poorly skilled, low productivity, workforce) seems just as relevant to the Ugandan health system. And her proposal that such change requires a 'multi-level regulation' apposite.

Pfister (2012) similarly approaches capabilities from the perspective of European citizenship. Pfister suggests that Sen's 'capabilities approach', or 'CA', places important emphasis on individual freedom. He suggests that this can be further developed to situate individual freedom within strong relational and political elements:

[The capabilities approach] is a powerful tool to put individual circumstances in the broader political, economic and social context (2012: 241).

Pfister emphasises the importance of context and differentiated needs to an understanding of the relationship between resources and functionings (or 'opportunities' and 'behaviour' in the COM-B model):

Differences between persons with equal resources in achieving functionings should not be explained with purely individual intermitting variables, such as (lack of) ambition, responsibility or (wrong) personal choice. [Rather we should be attentive to] differences in the capabilities of people to turn resources into functionings (2012: 242).

Capabilities then are not a given; they are a product of the interaction of resources (opportunities) with agency. The CA perspective shares Barbard's emphasis on the role of the State (or of citizenship) in providing citizens with the 'positive ability' and encouragement to participate in society (or their own workplace) and invest in their own social capital and training. The concept of 'agency freedom' (the freedom to achieve whatever the person, as a responsible agent, decides he or she should achieve) is then introduced and linked to the notion of 'goals':

We not only have to be able to achieve a goal but should be genuinely free to decide whether we actually want to achieve it or find an alternative 
objective (even one which others might view as less beneficial to our well-being) (Pfister 2012: 243).

Pfister suggests that the CA approach does not go far enough in terms of shifting the emphasis from the 'individual' (Homo economicus) to the person as 'situated self' and the fundamental importance of context to an understanding of human interaction and relationships. He suggests that the CA approach underplays the importance of power relations and the 'positional' quality of relationships and 'struggles'. Pfister's comment that 'even entitlements to certain capabilities have to include a notion of who is responsible for providing them' (2012: 249) immediately resonates with our empirical work in Uganda, placing an emphasis not only on the individual health worker but also on their employing organisation or the State (Ministry of Health) to ensure that salaries are paid with some predictability (which they are not) and equipment and consumables provided. In this context, should the failure to remunerate be conceptualised as a simple lack of 'opportunity' as in the COM-B model or as a denial of fundamental rights (and disempowerment)?

The focus on citizenship in Pfister's paper reminded us of the (taken for granted) importance of the relationship that individual citizens have with the State in Western democracies. Citizenship, as a form of social contract, is what binds individuals to the state and vice versa. The concept is used extensively in debates about European social policy to frame discussions about agency, marginalisation and exclusion. How relevant are these debates to an understanding of malfunctioning health systems in Uganda? Might it be, for example, that the quality of these relationships, between Uganda as a State and its citizens, is a factor at least partially explaining the apparent 'demotivation' of healthcare workers? Indeed, could some of the behaviour characterised as demotivation or disinterest (or even limited individual capacity) be otherwise conceptualised as 'social struggles on the ground.' A form of civil disobedience: perhaps a rational response to marginalisation and oppression? (2012: 244). Or, what Kostakopoulou refers to as 'tactical subjectivity' (cited in Pfister 2012: 245).

It is interesting to take a step back here and consider the role that the National Health Service (NHS) plays in the UK not just in the provision of health care but also in symbolising the relationship between citizens and the State. The NHS (in common with the Ugandan health service) is 
a universal public service, free at the point of use. However, for the time being the NHS remains the sector of choice for the majority. The Ugandan system, on the other hand, is a residualised (ineffective) safety net for the poorest people who have no choice and no means to access private services. Even the majority of Ugandan healthcare workers would not use the services they provide. In this context, it is 'easier' to categorise the system and the patients who use it as 'other' or even as the 'undeserving poor' (Marshall 1950).

Environment is not then a disconnected or disembodied 'given' facilitating or obstructing individual choices in the public health environment. It is itself constituted through social interaction: 'law, political institutions, the economy and technology are always created, interpreted and endowed with meaning through social interaction [...] they are human constructions influencing our agency' (Pfister, 248).

We have noted that this work is primarily theoretical. However, it is interesting to note Pfister's reflections on the methodological implications of these approaches. He suggests that the majority of 'empirical operationalisations' of the CA approach are quantitative, 'drawing on aggregative data, indices and statistics' (p. 25l) and necessarily retrospective. In conclusion, he advocates complementing CA with 'qualitative interactionoriented' dimensions to 'investigate human beings as creators of the world they inhabit' (p. 252).

The work reviewed earlier introduces a variety of complex concepts to an understanding of health worker behaviour. What is most interesting is the weighting attached to context and the emphasis on employment and the quality of work. The clinical focus and epistemology underpinning the behavioural science approach has the (unintended) effect of drawing us away from the obvious: that understanding the behaviour of health workers in low-resource settings is fundamentally about work and not health as such. Moving on from the concept of capabilities, we also began to feel uneasy about the (apparently highly individualised) concept of 'motivation' in behavioural science and how it sat alongside our experiences of Ugandan health workers. Coupled with the emphasis on labour markets in Barbard's paper, this led us to return to and widen our knowledge of the literature on HRM or the more specialist sub-field identified as 'human resources for health.' 


\section{Finding Commonality? Perspectives from Human Resource Management (HRM)}

What the world wants is a good job. That is one of the biggest discoveries Gallup has ever made. It is the single most dominant thought carried around in the heads of most people ... it establishes our relationships with our city, our country, and the whole world around us (Clifton 2007: 3).

Social science, and perhaps research in general, has a tendency to seek out the abnormal or exotic unintentionally glossing over the mundane everyday commonality that unites rather than distinguishes us. This quote from a Gallup survey underlines the most obvious but often overlooked issue that lies at the core of health worker motivation. It does not provide all the answers or angles but it presents a sound universalising, rather than essentialising, starting point from which we can begin to understand context and diversity.

There is a lively and burgeoning corpus of research focusing on health worker motivation from what can be loosely described as 'human resource management' (HRM) perspectives. Buchan, with reference to the UK context, argues that 'the importance of the management of human resources to the success or failure of health sector reform has often been overlooked' $(2000 ; 319)$. Interestingly a review of a large number of papers from a range of international contexts suggests a powerful commonality of 'drivers' in diverse low- and high-resource settings. The work by Nzinga et al. on the implementation of guidelines in Kenyan hospitals concludes that 'the barriers identified are broadly the same in theme to those reported from high-income settings' (2009: 1).

One of the things that distinguish the HRM approach from behavioural science is the concept of 'motivation.' As noted earlier, behavioural science tends to treat motivation as an intrinsic variable: internal to the individual. In a recent presentation on behavioural science approaches and their potential contribution to understanding behaviour change in global health, Byrne-Davis argued that 'when we as behavioural scientists talk about motivation it is very moment to moment $[\ldots]$ motivation [is about] automatic reflexes; about what we're going to do - a kind of in the moment reflex' (presentation to the THET Patient Safety Program meeting, November 2015). 
The HRM literature centrestages motivation but the key to motivation lies not with intrinsic individual attributes but organisational contexts: it is relational and in some contexts disproportionately extrinsic. In this model, the structural/organisational context lies at the heart not the periphery of behaviour change. The study by Nzinga et al. is interesting as it positions itself within the behavioural science spectrum as an example of evidence-based medicine (2009: 1). However, the qualitative approach utilised (interviews) generates a whole range of findings, nearly all of which are focused on local contextual conditions and weaknesses in HRM. It is perhaps no surprise that they conclude that, 'Future research might benefit from the disciplines of organisational management as well as behavioural sciences' (2009: 8).

The organisational focus characteristic of HRM theories tends to define motivation quite narrowly as 'an individual's degree of willingness to exert and maintain an effort towards organisational goals' (Dieleman et al. 2006: 2). The emphasis on the organisation is further exemplified in the definition of HRM offered by Mathauer and Imhoff: 'Human Resource Management is the management of people in an organisation' (2006: 4). Having said that, Mathauer and Imhoff (2006) take a broader view of motivational dynamics suggesting that 'Health workers are demotivated and frustrated precisely because they are unable to satisfy their professional conscience' (2006: 1). This latter approach certainly resonates better with our experiences in Uganda perhaps because of the sheer lack of effective and tangible HRM in most (dysfunctional) facilities. The views of the following Ugandan health worker suggest an identification of himself as a professional rather than as an employee (although the issues he raises pertain to wider aspects of resource management):

Poor working conditions means you have the knowledge but people are dying in your hands. Emotionally, professionally, you feel you should not be in that area. For us health workers we feel touched when these people die in our hands yet we know we could have saved their lives if $1,2,3$ things were in place. And we know it is possible to put them in place but somebody somewhere has not put them in place. So, as a professional, when people are dying in your hands because you don't have some of the things to help them and you know what to do - you feel you should not be in that place actually - you feel those people shouldn't come to you (UHW).

Khan and Ackers (2004) critique the 'unitarist' perspectives of what they term 'Western HRM' advocating a more pluralist approach capable of 'institutionalising some elements of the "African social system" into formal 
HRM policies and strategies' (2004: 1330). They are not referring so much here to multiple employment or systems within systems (i.e. corruption) but more to the role of 'normative stakeholders' such as extended family clans and religious brotherhoods. In the Ugandan context, tribal affiliation plays an important role both within existing organisations and as external, interlocking organisations. Tribal affiliations also compete with and lie in some tension with national and organisational identities. For now the main point here is that viewing motivation from the perspective of 'the' single employing organisation may be far too narrow.

The following section summarises some of the key findings reproduced in a selection of papers on health worker motivation. ${ }^{10}$ There is a strong tendency to distinguish financial from non-financial variables. Whilst, unsurprisingly, especially in low-income settings, pay is a key driver of health worker motivation, it is by no means the only or even the most important factor. Pay emerges most powerfully for less-well-paid cadres where pay is currently below subsistence level; it is impossible to maintain a basic quality of life through full-time public employment. This is the case in Uganda for most cadres of staff. A typical nurse or midwife in Uganda earns between 400,000 and 800,000 Ugandan shillings a month $(£ 100-£ 170)$ depending on levels of qualification. ${ }^{11}$ Length of service continues to have a major influence on pay in Uganda, despite its discriminatory consequences. ${ }^{12}$ This reduces incentives for individual investment in career development.

A further complication arises due to administrative inefficiency and poor financial governance resulting in health workers often not being paid at all for months. Doctors (and specialists) are generally less likely to stress pay as a key motivating factor no doubt because they are able to top-up their wages through private work (as noted in Chapter 2). The qualitative research by Mathauer and Imhoff on non-financial incentives acknowledges the importance of financial incentives, 'especially in those situations where income is insufficient to meet even the most basic needs of health professionals and their families'. [However] 'Increased salaries are by no means sufficient to solve the problem of low motivation ... More money does not imply more motivation' (2006: 2). Indeed, and unsurprisingly if we reflect on our own experiences as employees, ${ }^{13}$ issues like security, leadership, recognition, clear understandings of roles and workloads, equitable access to opportunities for professional and career development, equality, autonomy, participation and access to the resources essential for effective work (consumables, equipment and medicines etc.) are all frequently cited (Fig. 4.3). 
Lack of leadership (poor role models) and supportive management: lack of active engagement in development of organisational goals

Lack of collaborative and inter-professional decision making \& team working (hierarchy and disrespect especially from doctors) contributes to the development of a culture in which knowledge is not valued or shared (envy and isolation)

Overwhelming workloads and lack of workload management compounded by endemic absenteeism (see below).

Poor communication

Lack of recognition and appreciation (blame culture)

Absence of 'open recruitment' (meritocratic and transparent)

Lack of clear Role Descriptions (especially for task-shifting cadres)

Lack of Performance Review/Appraisal systems: lack of incentives and lack of disciplinary action/enforcement

Lack of career development/progression opportunities (career ladders)

Lack of /unfair access to continuing professional development resource

Poor working environment, working conditions, infrastructure (lack of equipment, consumables and drugs) due to resource shortages and endemic corruption

Fears about personal safety and health

The pervasive influence of systemic corruption at all levels

Daily challenges to professionalism; the inability to work effectively and witnessing the immediate consequences of that.

Fig. 4.3 Non-financial human resource variables influencing health worker motivation in low-resource settings (Source: Authors' summary of reviewed HRM literature)

There is insufficient scope in this chapter to discuss these components of HRM in any detail. A clear message emerges that the health systems crisis in many low-resource settings is conceptualised as a crisis in human resource management and not inadequate capabilities per se. Indeed, Mbindyo et al. conclude their study (in concordance with BehSci theories) with the following statement:

Interventions that aim to change worker practice simply by offering training are likely to fare poorly unless attention is paid to those factors influencing the motivation of health workers to change and perform well at individual, organisational and system levels (2009: 9). 
According to the HRM model, the quality of the work environment (i.e. extrinsic factors) determines health worker motivations and poor performance/outcomes. It is the lack of congruence between individual goals (which may derive from their training, education, experience and professionalism), the goals of the organisation they are employed by and their ability to achieve these goals that impacts motivation.

From a methods point of view, it is interesting to note that the HRM work reviewed here is quite eclectic, often drawing on multi-disciplinary perspectives. And, most of the papers reviewed are based on in-depth qualitative research in the field. Mbindyo et al., for example, argue that qualitative methods are necessary 'to explore the depth, richness, and complexity of staff motivation’ (2009: 2).

\section{Perspectives from Evolutionary Economics}

At this point in our 'journey' we came across, quite by chance (or serendipity), another body of work. We had in fact worked in collaboration with Carolina Cañibano, ${ }^{14}$ an economist, for some years (on research careers) and it was while searching for a paper linked to that work that we came across a publication with the term 'capabilities' in the title. On initial reading we realised that, despite the complexity of its theoretical underpinnings ${ }^{15}$ and language and entirely different context, this new area of work (to us) had surprising relevance to the work we had been doing in Uganda.

Their work is presented as a critique of economic theories, including neoclassical and neo-Darwinian theories which fail, in the authors' views, to capture the role played by 'purposeful human action'. The same critique could be levelled at the concept of 'automatic behaviour' represented in the COM-b model.

Along with most of the evolutionary economics literature and the contributions to the discussion on capabilities from organisational and management studies, this work is targeted on innovation processes taking place within organisations with an implicit focus on the context of the 'firm' (i.e. private, market-oriented, for-profit, organisations) and economic systems change. One might question the relevance of this framework set within the context of 'knowledge economies' to an understanding of the role of professional voluntarism in public health systems in Uganda. At face value, the diversity of context and language may suggest that opportunities 
for policy transfer are minimal. Again, a systematic review would have screened this kind of work out.

However, if we can see beyond this diversity, core commonalities emerge.

With reference to scientific mobility, Kuvic defines 'productivity' and 'innovation' in a way that appears immediately relevant:

Productivity is about the quality of work produced. Innovation entails specialised knowledge or creativity that is 'less easily measured' and the output is also based on the level of motivation of the individual. It is essential that employers build an environment in the workforce that can foster this type of work (2015: 16).

If we take the view that low-resource settings (such as Uganda) are also part of the global knowledge economy, conceptualise health systems change in terms of innovation and health workers as economic actors (within these systems) and the logic of understanding health worker outcomes in terms of 'productivity', then the relevance is clear. Indeed, we would argue that using this form of language allows us to avoid the risks of essentialising health worker behaviour in low-resource settings (capabilities and motivations) and emphasising the core commonalities between firms in high-resource settings and public health organisations (in both high- and low-resource settings). The language of innovation and entrepreneurship is also far more positive and aspirational.

The following section distils some of the key concepts and processes represented in the work of these economists enabling us to identify the links with the theories discussed earlier and the potential for more holistic, multi-disciplinary, theoretical underpinnings to support the deployment of professional volunteers in health systems change.

Muñoz et al. (2011) propose an 'action plan framework' to focus on the theoretical concept of intentionality. Intentionality, that is, the dynamics of goals formation towards which agents direct their action, plays a major role in driving economic change. In pursuing their intended goals agents activate learning and the potential for new knowledge combinations emerges. This is the key mechanism for the evolution of capabilities. In addition, goals may be of different sorts. Agents (read health workers) may conceive highly transformative goals and direct their action towards new imagined realities but they may also formulate action plans with poor transformational potential. To 
account for this qualitative difference between types of action goals, the authors define 'innovative intentionality' as 'the will to conceive or imagine realities with the purpose of making them effective' (Cañibano et al. 2006: 319). Economic agents, as individuals or organisations, can in turn be qualified as operating with higher or lower levels of innovative intentionality. This approach and the weighting given to intentionality as the key driver can be contrasted both with the behavioural science model and, as Muñoz et al. explain, with the economic literature which, 'argues that knowledge is the only foundation of capabilities' (2011: 194). For Muñoz et al., economic evolution (systemic change) does not come about as a result of the growth of knowledge per se. Intention, not capability, is the starting point.

The concepts that immediately captured our imagination and resonated most starkly with our experience in Uganda included the explicit engagement with agency and the emphasis the model places on existing knowledge. Learning is not something that happens to people as passive actors/ victims (or empty vessels) but something that is essentially relational, interactive and cumulative. The individuals concerned are not devoid of (or lacking in) knowledge per se; indeed, it is their experiential (tacit and highly contextualised) knowledge - 'their perceived realities' - that shapes their response to new learning and imported knowledge. Agency is explicitly recognised in this model, which assumes that 'humans have sufficient intelligence and incentives to anticipate and avoid the selection effects' associated with evolutionary, Darwinian theories (Muñoz et al. 201 1: 194 citing; Witt 2004: 128).

This approach to intentionality helps us to see motivation as contextualised and as much about extrinsic as it is about intrinsic factors. In the first instance, health workers are heterogeneous and this heterogeneity is 'not only a matter of differences in knowledge [or innate ability], but also of differences in action goals and intentions' (Cañibano et al. 2006: 319) and their subjective responses to means (opportunities) or plans will shape their approach to new learning. Furthermore, a health worker's motivation will vary over time and space and in relation to diverse plans. A person, according to this model, cannot be placed on a linear motivation-demotivation or innate intelligence continuum: their degree of motivation will necessarily vary according to specific plans (places, conditions and relationships). Seeing motivation as fundamentally contextdriven helpfully removes some of the essentialising (and potentially racist) elements of the behavioural science model. 
Although Muñoz and Encinar (2014a) locate their paper within the paradigm of innovation systems and speak of knowledge-based economies and 'firms' their point that 'the systemic properties of the system emerge as a result of agent interaction' (2014a: 72) appear just as relevant to an understanding of public healthcare systems. The system is as it is (and is subject to change through) the interaction of agents operating at multilevels (from the Ministry of Health, through District Health Authorities, tribal boundaries and down to health facilities and individual health workers employed within them).

Whilst similar concepts are identified in the work discussed before, the 'action plan' model places unique emphasis on intentionality (the intention to do something) and conceptualises it as part of action planning or goal setting. It is this intentionality and planning that drives the process and activates learning and not the other way around (albeit in a constantly iterative and reflexive process).

The (subjective) process of planning so central to this approach is informed by deeply localised knowledge of the opportunities and constraints open to individual agents. The concept of 'information stocks' by Muñoz et al. (2011: 198) captures for us the importance of recognising pre-existing contextualised knowledge as knowledge and not simply lack of interest/ demotivation. This may take the form of explicit, experiential knowledge (of delivering countless dead babies, for example ${ }^{16}$ ) and/or tacit knowledge (defined here as know-how, ${ }^{17}$ skills, competencies, routines, capacities, capabilities' (Muñoz et al. 2011: 312).

Rather than the lack of knowledge it could be the sheer weight (grinding heaviness) of tacit knowledge that influences attitudes to new learning. Arguably, it is precisely this knowledge that newly arriving professional volunteers lack, limiting their own ability to formulate innovative knowledge re-combinations that restrict progress. It is not to say that the cutting-edge clinical expertise they bring is not of value but needs time (and humility) to settle and recombine with local knowledge in order to identify effective and sustainable and contextualised interventions. As Williams and Balatz (2008a) assert, this kind of knowledge travels less easily and requires co-presence.

This model doesn't privilege new learning and helps to explain how new learning (introduced by professional volunteers) must be seen to work in combination with existing knowledge (rather than displacing it). Echoing the work of Williams and Balatz (2008a) and Polanyi (1959), Muñoz et al. suggest that a significant proportion of organisational and 
individual knowledge is tacit (2011: 312) and yet when professional volunteers and development organisations intervene in low-resource settings they tend to focus on training in explicit (clinical) skills and, in the process, assume that the failure to behave in certain ways is a reflection of the absence of knowledge rather than the presence of it and its impact on health workers' perceived reality.

'Action planning' is described by Muñoz et al. as the 'analytic unit connecting means/actions and goals' (2011: 195). And connections play a very central role in this model: 'Agencies (individuals and organisations) make plans and planning implies making connections' (p. 196). It is the 'recombinant process of connections' that may generate what the authors term 'novelties'. ${ }^{18}$ The concept of connections here goes beyond a narrow definition of connections as social capital (networks and contacts) to embrace the boundary spanning / brokering qualities of knowledge itself. This fits with the authors' assertion that 'learning consists of testing (and eventually retaining) new connections that prove useful for agents to reach their goals' (p. 196).

Furthermore, imagination plays a central role in action planning. The concept of 'imagined realities' resonates powerfully with our work with Ugandan health workers and captures perfectly the dynamics of their highly contextualised situations. Is it possible in the context within which Ugandan health workers are placed to imagine a different reality?

Muñoz and Encinar (2014b) point out that economic processes (systems) are historical and action planning by agents takes place in a context of 'radical uncertainty' (p. 319). In that sense planning is not an objective, linear, exercise but a reflexive, subjective and uncertain process.

The fundamentally subjective quality of the concept of perceived/ imagined realities, coupled with the acknowledgement of existing deeply experiential and localised knowledge (as knowledge rather than the absence of it), enable us to understand the equally important concept of 'bounded rationality' (Simon 1985; Jones 1999) employed by these authors. Behaviour (or the lack of it) that may be interpreted by the outsider observer (a professional volunteer, for example, or clinical 'expert') as irrational or unproductive may, when understood in context, be rational (a new reality may not be possible).

The 'action plan' model identifies the importance of 'means,' conceptually equivalent to the 'opportunities' represented in the behavioural science model, and an implicit component of context and perceived realities. Conceptually it is present but underspecified in what is essentially 
a theoretical model. This is an area where the HRM research coupled with our own contextual knowledge can add flesh to the conceptual bones (framework). The fact that the economics model was not specifically designed to 'fit' the context to which we are now seeking to apply it perhaps explains this lack of specificity which, in the case of the Ugandan health system, will have profound effects on its application. ${ }^{19}$ This is captured poignantly by Muñoz et al. as a 'dialectical dance between feasibility and desirability' (Muñoz et al. 2011: 198). Health workers, as agents, evaluate and formulate their plans in the context of their own experience of what works.

Cañibano et al. (citing Teece et al. 2000) define 'dynamic capabilities' as 'the ability to reconfigure, redirect, transform, and appropriately shape and integrate existing core competences with external resources and strategic and complementary assets to achieve new and innovative forms of competitive advantage' (2006: 313). Furthermore, the process of acquiring or developing dynamic capabilities is a 'collective learning process from which an organisation improves its ability to achieve its goals' (p. 313).

The reference here to learning as a collective process is interesting from the perspective of professional voluntarism and health partnership activity where learning, whether in the classroom or in one-to-one mentoring, is almost always seen and planned as an individual process (taking person $\mathrm{X}$ from point $\mathrm{A}$ to $\mathrm{B}$ along a continuum of measurable (quantifiable) learning outcomes. It would be interesting to assess whether developing more active forms of learning focused on collectives $^{20}$ (multi-disciplinary teams within facilities) and working with their imagined realities may activate learning and improve outcomes.

It is the interaction of intentions and action goals that drive the evolution of 'dynamic capabilities'. Capabilities in this model are certainly not reducible to genetic (inherited) capacities (such as intelligence). Rather they are informed by existing knowledge and constantly reshaped.

Intentionality linked to goals is what 'activates the development of capabilities, the testing of new connections within a system, and, therefore, the generation of new knowledge' (Muñoz and Encinar 2014a: 75).

The 'action plan' model presents learning as a (hyper-)active process involving the 're-combination" 21 of new knowledge with existing explicit and tacit knowledge. Muñoz and Encinar describe intentions as 'triggering $[\ldots]$ driven learning processes' configuring connections which give 
rise to evolving capabilities. (2014a: 75). In the language of evolutionary economics, these may stimulate 'entrepreneurial experimentation'. The reference to the creation of 'new genetic material' (p. 76) represents an interesting challenge to the more essentialising tendencies of behaviour science and classical economics, which assume that genetics are fixed and determine capabilities (or are the same thing).

Ultimately, innovative intentionality is the activator of constantly and reflexively evolving 'action plans' that interact with 'social reality' to drive transformative change. Of course, such change is not always wholly or even partially effective: the authors accept the possibility that planned action may fail to lead to intended outcomes as a result of unintended consequences or externality effects (Muñoz and Encinar 2014b: 318).

As noted earlier, this area of work is entirely driven by theory and remains untested in any empirical context. This in part explains its luxuriousness and ability to deal with complexity. ${ }^{22}$

\section{A Synthetic, Multi-disciplinary Approach?}

Echoing the conclusions of Nzinga et al. (2009), Muñoz et al. conclude that Understanding human behaviour lies at the 'frontier of economics and psychology' (2011: 317 citing Brocas and Carillo 2004). Indeed, all of the authors reviewed here advocate the need for multi-disciplinary approaches even if they themselves resist the challenge. Having reviewed the approaches in the context of our much grounded action-research on professional voluntarism, we feel that the model that best fits the situation is that represented by the SI Framework.

We are particularly attracted by the attention the economic 'action plan' approach pays to existing knowledge or 'information banks' and the effect this knowledge has on perceptions of what reality is and could be. We feel that this theory helps us to understand why so many interventions fail. Knowledge does not activate change: rather intentions organised through plans (and agency) drive and activate learning. And new learning builds connections between people and knowledge to create the conditions in which change can begin to be imagined and actioned. The emphasis on the collective and connected quality of active learning is also very helpful and encourages us to see beyond learning as an individualised process. 
Having said that all the models share broad ideas, each one discusses individual behaviour in some form of structure-agency/choice-constraint framework. Each recognises the importance of tacit knowledge. Each refers to motivation and to goals or plans in some shape or form and to opportunities or resources and each pays some attention to multi-level contexts. The capabilities approach is focused primarily on supranational and national systems using the concept of citizenship to describe the relationship between individuals (as citizens) and the state. The state is important but we must see this as multi-level too and, in the Ugandan case, transected by other critical elements of identity, belonging and political affiliation: tribes remain of great importance, brokering the kinds of citizenship relationships identified in the European Union. Schaaf and Freedman's work on health worker posting emphasises the importance of recognising that the 'state' is not a unified or necessarily benevolent actor but reflects many conflicting interests and norms that shape individual and organisational behaviour (2015: 7).

With the exception of HRM, all of the approaches tend to gloss over the organisational context, which plays a vital intermediary function. Only HRM theory really captures the everyday reality of employment relations and the quality of work. However, it tends to perhaps overemphasise 'the organisation' and the impact of employer-employee relations on wider motivational dynamics. Many employees in Uganda and elsewhere will move between employers or (as is very common) have more than one job. The reference to professionalism illustrates the notion that a person's identity and sense of responsibility may not always align first and foremost with a particular employer. Indeed, doctors in Uganda (and elsewhere) often identify themselves with their profession more than a specific health facility that pays what is in most cases a tiny fraction of their overall income. International organisations (NGOs and health partnerships) may also be important organisational actors here perhaps interfacing with local employing organisations (such as the SVP) or directly with individual health workers (through salary top-ups or moonlighting).

Certainly it is widely recognised that individual employers play only a partial role in contemporary career planning. Perhaps reflecting its empirical strengths (and grounding in qualitative research) the HRM literature is important to our work not so much because of its theoretical contribution but the attention to detailed analysis of employment quality that resonates so sharply with our own understanding of Ugandan health worker's 
experiences. This level of analysis has supported our ability to design very practical evidence-based interventions.

This concept of bounded rationality (in the economic model) is perhaps far more relevant than the architects of these papers anticipated. One of the limitations of the papers, perhaps reflecting the level of abstraction, is the lack of attention to conflict and power: the possibility of multiple realities and parallel organisational cultures. Corruption is systemic in Uganda especially within the public sector; it can be described as a culture. It is pervasive and starts from the very top of organisations and the systems within which they are based and operates through powerful, organised syndicates. Health workers (and patients) are acutely aware of its existence, the personal benefits that derive from it and the profound risks associated with challenging it. It is interesting to note the reference by Muñoz et al. to the entrepreneur as a 'destabilising agent' (2011: 199). 'Destabilising' in this context could imply creative disruption triggering innovation (in the right direction). Alternatively, or simultaneously, it could refer to the impact of corruption. This detailed (tacit and explicit) knowledge of how corruption works at every level and in every decision necessarily shapes both imagined realities and action plans. In this sense, there may be two parallel systems operating in marked tension with each other within a health facility or authority. Chapter 3 has discussed the impact of corruption on professional volunteers and their relationships with Uganda health workers identifying the dynamics of power and hierarchy (positionalities). None of the theories reviewed pays explicit attention to these dimensions of context. Interestingly it is often the lack of knowledge on the part of foreign agencies and individual volunteers rather than their superior clinical knowledge that limits impact and generates unintended consequences. ${ }^{23}$ Of course, corruption pollutes not only organisations but also the wider system that nurtures it, fundamentally weakening a sense of identity with the state at national or local level and also with 'leaders' (at every level).

Whilst we can identify closely with the concept of 'action planning' as a vehicle for the exercise of individual agency based on the recombination of disparate knowledges, we have some concerns that the emphasis in the material reviewed, perhaps reflecting the business/private sector context, fails to explain inaction or stasis. Or, situations when human action, qua rational, as Muñoz and Encinar (2014a: 75) put it, could amount to non-decisions or inaction. On a practical level, it may prove impossible to imagine returns on an investment (in training, for example, 
or even coming to work regularly). Equally (and commonly) the risks associated and predicted (as a component of perceived reality) may lead a person to consciously decide not to challenge a corruption syndicate or even show some initiative. Indeed, we have seen a number of innovative individuals motivated to formulate plans which activate new learning and bring about systems change effectively punished and threatened by their peers and their line managers for stepping out of line. Simply being seen with 'muzungus' leads to perceptions of financial gain and ensuing envy followed by punishment. Just as the association of 'immobility' with competitiveness has been challenged (Ferro 2006), inactivity needs to be seen as rational in certain contexts.

\section{CONCLUSION}

The research review work that formed the basis of this chapter was entered into following our growing realisation grounded in evidence gained from in-depth qualitative research, that many if not most of the interventions utilising professional volunteers as knowledge brokers have largely failed to generate visible and sustainable impact on health systems. Or, put more simply, training Ugandan health workers has failed to translate into evidence of individual behaviour change. The exploration of other research marked an attempt to answer the 'why' question and, if necessary change our methods of intervention.

All of the approaches reviewed here offer important insights and we would not wish to privilege any disciplinary or theoretical approach but rather to identify those aspects that we feel are most applicable. Recent studies applying the COM-B model to an evaluation of behaviour change in CPD interventions in low-resource settings concluded that 'None of capability, opportunity or motivation were found to predict either behaviour or behavioural intention' (Byrne-Davis et al. 2016: 68). The authors suggest that this may reflect the fact that the validation of these tools took place in 'resource-rich, high-income environments' which may 'reduce the applicability of some behaviour change theories to this [low resource] context'. Having said that, the other theories reviewed here have not attempted empirical verification remaining at 'ideas' stage.

Our empirical work confirms the importance of the three components of the COM-B approach: namely of capabilities, opportunities and motivations. However, it rather turns the equation on its head 
suggesting (as SI theory proposes) that intentionality comes first: you have to be motivated to learn. And this motivation is not intrinsic but, and especially in low-resource settings, is fundamentally extrinsic and context bound. And, in that frame, local knowledge, especially tacit knowledge of what works and can even be imagined to work, drives motivation. So knowledge can act as a break on individual intentions (if we have learnt repeatedly that plans or interventions do not work or cause us personal risk/harm).

Chapter 5 builds on the material presented in this and previous chapters to reflect on the learning that has taken place since we began to deploy professional volunteers to Uganda. Perhaps unusually at this stage in a book, it organises this reflection around two empirical case studies. These are used to illustrate the iterative quality of our evidencebased interventions and the role that research has played in taking us to the conclusion that the primary learning is learning from failure and not quantifying success.

\section{Notes}

1. Serendipity or happenchance has been increasingly recognised as important sources of social capital in research (Ackers and Gill 2008).

2. Epistemology is defined in the Oxford Dictionary as 'the theory of knowledge, especially with regard to its methods, validity, and scope, and the distinction between justified belief and opinion'.

3. COMb stands for Capabilities, Opportunities, Motivation and Behaviour.

4. www.vichealth.vic.gov.au/cochrane.

5. An example of how this approach can be applied to the evaluation of health partnership interventions can be seen in Jones et al. (2013).

6. The authors then developed a complex scientific tool incorporating a Delphi-style consensus process and a range of 'open and closed sort tasks' and 'fuzzy cluster analysis' as the basis for the refinement of the TDF.

7. This definition of motivation as 'processes in the brain that energise and direct behaviour' is taken directly from Robert Wests' PRIME Theory (2006). It is important to point out that PRIME theory was developed in the context of alcohol and drug addiction in the UK and not health worker behaviour.

8. See Guardian http://www.theguardian.com/politics/2013/apr/08/mar garet-thatcher-quotes.

9. This is the title of a journal which captures a lot of this material. 
10. As noted, the objective of this chapter is not to present a comprehensive literature review across all disciplines. Our aim has been to identify work which we feel 'speaks to' our objectives and has the potential to contribute to the evidence base informing our interventionsevidence base informing our interventions. This section reviews the following papers: Buchan (2000: 2004); Chen et al. (2004); Chopra et al. (2008); Dieleman et al. (2006); Franco et al. 2002); Mangham and Hanson (2008); Mathauer and Imhoff (2006); Mbindyo et al. (2009); Nzinga et al. (2009); Stringhini et al. (2009); Willis-Shattuck et al. (2008).

11. Our estimation of the level of income required in Kampala to meet basic subsistence needs (housing, food and school fees) for a family with two children is around 2 million Ugandan shillings (about $£ 500$ a month).

12. Seniority-based pay is explicitly prohibited as discriminatory under European Union employment law.

13. Similar findings came out of a study on pay and remuneration in research in the UK (Ackers et al. 2006).

14. We are very grateful for the opportunity to present a version of this chapter to Dr Cañibano's group in Valencia and to have received her insightful comments on this chapter.

15. Helfat and Peteraf (2009) suggest the level of complexity in the SI framework may have generated some confusion even within the field.

16. Ugandan health workers will have considerably more hands-on experience of many obstetric complications than their professional volunteer counterparts.

17. Gebauer refers to this as 'procedural knowledge' as distinct from 'declarative knowledge' (2012: 59).

18. The concept of 'novelties' is new to us and at first seemed rather strange. If we understand this as meaning new ideas or innovations it works well in the context of our work. Muñoz and Encinar define novelties as 'new realities' (2014b: 319).

19. Muñoz et al. do refer specifically to monetary and non-monetary elements of action plans echoing a core distinction in the HRM literature (2011: 195).

20. The concept of the 'collective understanding of knowledge' through 'assimilative learning' is also referred to by Gebauer et al. (2012).

21. This echoes Williams and Balatz's (2008a) reference to knowledge combinations discussed in Chapter 2.

22. Muñoz and Encinar point to criticisms that the SI approach is in fact 'overtheorised'.

23. In practice, this is a difficult but necessary component of induction processes and may be a factor to take into account when considering the efficacy of length of stay. 
Open Access This chapter is distributed under the terms of the Creative Commons Attribution 4.0 International License (http://creativecommons.org/ licenses/by/4.0/), which permits use, duplication, adaptation, distribution, and reproduction in any medium or format, as long as you give appropriate credit to the original author(s) and the source, a link is provided to the Creative Commons license, and any changes made are indicated.

The images or other third party material in this book are included in the work's Creative Commons license, unless indicated otherwise in the credit line; if such material is not included in the work's Creative Commons license and the respective action is not permitted by statutory regulation, users will need to obtain permission from the license holder to duplicate, adapt or reproduce the material.

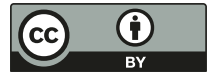

